\title{
CLINICAL AND EPIDEMIOLOGICAL PROFILES OF NOVEL VIRUSES IN LOWER RESPIRATORY TRACT INFECTIONS IN CHILDREN
}

\author{
Alexandru-Ioan Ulmeanu, Carmen Zapucioiu, Coriolan-Emil Ulmeanu \\ Pediatrics Department, "Carol Davila" University of Medicine and Pharmacy, \\ ,, Grigore Alexandrescu“ Emergency Children's Hospital, Bucharest
}

\begin{abstract}
Viral respiratory tract infections are one of the leading causes of morbidity and mortality in pediatric pathology. The authors provide the latest information on the structure, pathogenesis, clinical features, epidemiology and response to treatment of newly discovered viruses affecting the respiratory tract in children: bocavirus, metapneumovirus, coronaviruses, rhinovirus $\mathrm{C}$.
\end{abstract}

Keywords: bocavirus, metapneumovirus, coronaviruses, rhinovirus $\mathrm{C}$, pneumonia, bronchiolitis

\section{INTRODUCTION}

Viral infections of the lower respiratory tract represent an important cause of morbidity and mortality in pediatric pathology. Every year in the pediatric population, worldwide, are recorded approximately 100 million cases of viral pneumonia, representing $60 \%$ of lower respiratory tract infections (LTRI) of the child. The viruses most frequently involved in LTRI are respiratory syncytial virus (RSV), rhinovirus, metapneumovirus (hMPV), bocavirus (HBoV) and parainfluenzae virus. They can be isolated alone or they can be diagnosed in viral coinfections. $30 \%$ of the children with viral pneumonia have evidence of bacterial infection at the time of diagnosis (1). In addition to well-known viruses involved in children's LTRI, important and highly topical information appeared in literature about clinical and epidemiological characteristics of new discovered viruses that can infect the respiratory tract of children: bocavirus, metapneumovirus, coronavirus and rhinovirus $\mathrm{C}$.

\section{BOCAVIRUS}

Bocavirus (HBoV) is a recently described virus, discovered in 2005 in nasopharyngeal aspirates of swedish children. The prevalence is about $10 \%$ overall and it is frequently involved in infections of upper and lower respiratory tract in children, which may complicate with severe respiratory distress especially in premature infants. It is one of the most common respiratory viruses detected under the age of 5 years with a maximum of frequency between 6 and 24 months. The prevalence in the adult population is very low. $(2,3)$

\section{Viral structure}

Bocavirus is part of the Parvoviridae family, subfamily Parvovirinae, genus Bocavirus. It is a small, non-enveloped, icosahedrical virus with linear, single-stranded DNA genome. The capsid is composed of two viral proteins VP1 and VP2 and is very similar to parvovirus B19 (4). There are currently four bocavirus species described: HBoV1, 
HBoV2, HboV3, HboV4. The first subtype is involved in respiratory tract infections but can be isolated also in faeces, while the last three were isolated mainly from the stool.

\section{Mode of transmission}

Bocavirus is a ubiquitous virus whose transmission mode is similar to parvovirus. It is transmitted mainly by inhalation of airborne droplets or by contact with infected secretions. Most studies show that $\mathrm{BOV}$ infection occurs throughout the year, with peak incidence in winter and spring. $(5,6)$. Most children are infected by the age of six, antibodies directed against the capsid are formed and because of the lack of variation in the viral envelope they provide long-term protection. Adults most commonly present with antibodies demonstrating that the infection is more common in childhood $(7,8)$.

\section{Pathogenesis}

Bocavirus infection leads to the destruction of the epithelium of the respiratory system by affecting tight cell junctions, with loss of cilia and hypertrophy of epithelial cells (9). During the acute infection DNA HboV,IgM-HboV can be detected in the serum which is indicative of systemic infection. Viral infection induces an immune response involving virus-like cytokine secretion by Th1 and Th2. The virus can persist in the body for a long time, even 4-5 months, most likely through persistent replication and elimination. The prolonged persistence could explain the high frequency with which the virus is involved in viral co-infections. $(10,11)$

\section{Clinical and laboratory features}

$\mathrm{HboV}$ was reported to be associated with respiratory infections like: common cold, viral-induced wheezing, asthma, acute bronchiolitis, pneumonia or acute otitis media. (12). In order of frequency, cough, fever, runny nose, wheezing and respiratory distress were the most common symptoms. Acute diarrhea, acute conjunctivitis, vomiting and rash were less frequently encountered. The occurrence and severity of symptoms has been associated with high levels of viremia $(2,13)$. Infections with other viruses cannot be differentiated clinically from bocavirus infection, however it was found that hypoxia and neutrophilia were encountered more frequently in HboV infection rather than RSV infection. (12) WBC count was normal, without evidence of inflammation but with the advent of neutrophilia. (14) The most common radiological appearance was of acute interstitial pneumonia with bilateral linear opacities, hyperinflation and atelectasis. There where no pathognomonic aspects (12).

\section{Diagnosis}

Most studies have used RT-PCR for diagnosis, but this determination is limited by the old and persistent infection. Thus, for a positive diagnosis is required to determine DNA HboV together with the presence of serum IgM antibodies in serum, or the dynamic 4-fold increase of IgG antibody titers together with the presence of symptoms (2). Were positively associated with certain viral infection: monoinfection, high viral load and the presence of viremia. Since in practice, antibodies or viremia determinations are difficult to perform, we can consider a certain diagnosis for infection a PCR determination with $\mathrm{HboV}>10^{4}$ genomes/ml.(2)

\section{Treatment}

Lower respiratory tract infections with bocavirus are self-limiting and usually uncomplicated, requiring only supportive treatment. Prednisolone was not effective in a group of children with wheezing and bocavirus infection $(15,16)$.

\section{METAPNEUMOVIRUS (HMPV)}

HMPV was discovered recently, in 2001 , by a team of dutch researchers in a group of children presenting with acute respiratory disease, from simple common cold to acute bronchiolitis and severe pneumonia. Studies in the literature suggest that HMPV has been involved in respiratory tract infections for more than 50 years. HMPV infection prevalence varies between $4-16 \%$ of patients with respiratory tract infection $(17,18)$.

\section{Viral structure}

HMPV is part of the paramyxoviridae family, Pneumovirinae subfamily, genus Metapneumovirus and can be found in two main forms: HMPV type A and type B. HMPV structure is very similar to RSV, which is also a member of the Pneumovirinae subfamily. HMPV is an enveloped virus, pleomorphic, with varying sizes and a RNA genome, related to avian MPV. Comparing HMPV genome to that of RSV was found that the structural proteins NS1 and NS2 are missing in the MPV, explaining the different types of immune responses encountered during the infection $(18,19)$. 


\section{Mode of transmission}

The mode of transmission is most often by inhalation of air droplets or by contact with infected secretions. Incubation is short, 3-5 days (20). Nosocomial infections have been reported. The virus is ubiquitous, but the variation is seasonal, with peak incidence in late winter and early spring in the northern hemisphere and late spring in the southern hemisphere. (21) It infects mostly children, most of whom are infected by the age of five, the peak incidence is 2 years old, reinfection can occur in adulthood due to obtaining an inadequate antibody titer after the first infection or by reinfection with a different genotype (22). HMPV infection can occur at any age, but it is more severe in young children and infants, and premature infants, those with chronic lung or heart disease and neuromuscular diseases, as well as those who have acquired the infection in the hospital setting. HMPV is common in viral coinfection, some studies suggesting that the association especially with RSV increases the severity of the respiratory disease. (18)

\section{Pathogenesis}

Beta1 integrin alpha-v facilitates penetration of respiratory epithelial cells (23). Infection leads to hypersecretion of mucus, hyperplasia of the epithelium and bronchial hyperreactivity. The bronchial hyperreactivity was proven by methacholine challenge test and emphasizes the idea that severe viral infections ofchildhood may be associated with subsequent development of asthma. (24)

\section{Clinical and laboratory features}

High viral load correlated with the presence of symptoms and disease severity in some studies (25). RSV coinfection influences disease severity in children under 2 years. (24)

The most common signs and symptoms found were cough, runny nose, fever and wheezing. The most frequent diagnoses encountered were acute bronchiolitis, viral croup, exacerbation of asthma, and pneumonia. (26)

In the initial stages of infection monocytosis with mild/moderate increases of C-reactive protein (CRP) were observed. In evolution, along with improvement of symptoms the WBC count normalized but with the maintenence of slightly increased CRP (18).

\section{Diagnosis}

RT-PCR is the most sensitive method for determining the hMPV. Imunofluorescent methods by
DFA techniques (direct fluorescent antibody) allow detection of the antigen in nasopharyngeal aspirates in 2-3 hours, but this technique is only available in specialized laboratories. ELISA serological tests that can detect the presence of antibodies against $\mathrm{N}$ protein can be used also for diagnosis (24)

\section{Treatment}

Treatment is mainly supportive, but ribavirin was effective in vitro against hMPV, reducing viral replication in infected mice. It has been used successfully together with non-specific immunoglobulins in a case of severe infection in a child treated with chemotherapy for Burkitt lymphoma (27) (28). Monoclonal antibodies, fusion inhibitors and siRNA (interfering RNA molecules) have shown promising effects in experimental studies (18).

\section{Prevention}

HMPV infection does not induce lasting immunity. The main problem encountered in designing a vaccine has been the difficulty in obtaining a strong and lasting immune response. There are currently ongoing phase 1 study to determine the efficacy and safety of a live attenuated vaccine against infection with HMPV (29). There are promising studies on laboratory animals showing efficiency of the monoclonal antibodies against HMPV when administered both before infection and after 48 hours from the installation of symptoms. $(30,31)$

\section{CORONAVIRUSES}

Coronaviruses are frequently involved in respiratory tract infections in adults but with an important role also in severe lower respiratory tract infections of the child. Members of the Nidovirus family they are divided into two major types: alpha coronavirus represented by HCOV229E, HCOVNL63 and beta coronavirus represented by HcoV- HKV1, HcoV-OC43 and MERS-COV and SARS-COV (32). HCoV are enveloped viruses with RNA genome that are very hard to grow on cultures. The first discovered strains in 1960 were 229E and OC43, in 2002 SARS was discovered and rapid after that the NL63 and HKU1 ubiquitous serotypes were described (33).

\section{Mode of transmission}

Transmission occurs most frequently by inhlation of air droplets but can happen also after contact with infected secretions. Serotype immunity is ob- 
tained after infection, but reinfection is possible. (34) (35)

\section{Clinical features}

The most common symptoms encountered during $\mathrm{CoV}$ infection were rhinorrhea, cough and wheezing. For subtypes 229E, OC43, NL63 and HKU1 the most common clinical picture is similar to rhinovirus infection (36). In particular HCOVNL63 was associated with viral croup and $\mathrm{HCoV}$ OC43 and HKV1 were isolated in infants with diarrhea and respiratory symptoms. HCOV can also be found in asymptomatic children (35). Coronaviruses have been isolated frequently in otic secretion of children with acute otitis media and have been implicated in severe exacerbations of asthma (36,37). Coronaviruses can cause severe nosocomial infections. $(38,39)$. Recent studies described a possible association of $\mathrm{HCoV}$ infections with multiple sclerosis and Kawasaki disease. (33)

\section{Diagnosis}

The diagnosis of infection is made by detection of the virus by RT-PCR in nasopharyngeal aspirate but can also be made with imunofluorescent techniques. Etiologic diagnosis is especially important for SARS epidemics, isolation of positive cases being very important in limiting the spread of the infection in the population. (33)

\section{Treatment}

Treatment is mainly supportive. In vitro studies show promising effects of chloroquine on coronaviruses but clinical trials are needed to confirm the results. There is no coronavirus vaccine approved for the infection. $(33,40)$.

\section{RHINOVIRUS C}

Rhinoviruses are the viruses most frequently involved in acute respiratory infections in adults and children, the child being the main reservoir of infection. $30-50 \%$ of upper respiratory tract infections are caused by rhinovirus. (41). Rhinovirus belongs to the Picornaviridae family. Three main rhinovirus species are known: HRV-A, HRV-B HRV-C with more than 100 serotypes. Rhinovirus $\mathrm{C}$ was discovered last, in 2007 and is a ubiquitous virus with similar effects compared to species A and $\mathrm{B}$ but was described to be more commonly involved in severe asthma exacerbations and complicated bronchiolitis of infants and children (42). It presents with different genome compared to spe- cies $\mathrm{A}$ and $\mathrm{B}$, is difficult to grow on cell cultures and the attachment receptor is different from ICAM1 but still unknown. Pleconaril, an antiviral that was effective against the species A, had no effect on HRV-C $(41,43)$.

\section{Mode of transmission}

HRV-C is found throughout the year but with peak incidence in autumn and winter, recent studies show that it is the rhinovirus most commonly encountered, accounting for about half of rhinovirus infections. The mode of transmission is most commonly by self inoculating infected secretions into the nose and conjunctiva. $(44,45,46,47)$.

\section{Clinical features}

Like HRV-A and HRV-B, rhinovirus $\mathrm{C}$ is involved most frequently in upper respiratory tract infections in adults and in children and is described as etiologic agent in acute otitis and sinusitis in children. About half of the child's asthma exacerbations are caused by viral infection and rhinovirus is involved in $50 \%$ of them. In particular Rhinovirus $\mathrm{C}$ is involved in severe exacerbations of asthma, severe bronchiolitis and in cases of recurrent wheezing more frequently than the other two species, and recent studies associates it as the causative agent in newborn and infant apnea (Apparently Life - Threatening Events) $(48,49,50,51)$. HRV-C can cause extrarespiratory infections being isolated from plasma and pericardial fluid of a 14 months child with severe pneumonia and pericarditis (52). Although in the past HRV were considered to be viruses involved only in mild and self-limiting infections with minimal impact on the health of the individual, with the discovery of HRV-C studies demonstrate that their role is even more important than RSV in lower respiratory tract infections of children in need of hospitalization and in triggering severe exacerbations of asthma and recurrent wheezing in children. $(49,53,54)$

\section{Treatment}

Treatment is mainly supportive, pleconaril the antiviral used for HRV A infection is not effective on HRV-C infection. (41)

\section{CONCLUSIONS}

Lower respiratory tract infections are one of the leading causes of infant mortality worldwide. New data continues to appear on newly discovered viruses that are promising and help us understand the 
genetic diversity, mechanisms of action and the way of defense and response of the human body during the infection. The knowledge of viral profiles help us to diagnose fast, accurate and treat correctly the respiratory tract infections of the children.

\section{REFERENCES}

1. Olli Ruuskanen, Elina Lahti, Lance C. Jennings, David R. Murdoch. Viral pneumonia, Lancet 2011; 377: 1264-75

2. Tina Ursic, Miroslav Petrovec. Human Bocavirus, Emerging Infectious Diseases, 2014, 191-201

3. B.D. Chow, F.P. Esper. The human bocaviruses: a review and discussion of their role in infection, Clin Lab Med, 29 (2009), 695-713

4. O. Schildgen, J. Qiu, M. Soderlund-Venermo, Genomic features of the human bocaviruses, Future Virol, 7 (2012), 31-39

5. Chia-Yunn Chuang, Chuan-Liang Kao, Li-Min Huang a, et al. Human bocavirus as an important cause of respiratory tract infection in Taiwanese children Journal of Microbiology, Immunology and Infection (2011) 44, 323e327

6. Chow B.D., Huang Y.T., Esper F.P. Evidence of human bocavirus circulating in children and adults, Cleveland, Ohio. J Clin Virol 2008; 43:302e6

7. Susanna K.P. Lau, Cyril C.Y. Yip, Tak-Iun Que, et al. Clinical and Molecular Epidemiology of Human Bocavirus in Respiratory and Fecal Samples from Children in Hong Kong, J Infect Dis. (2007) 196 (7): 986-993

8. K. Kantola, L. Hedman, T. Allander, et al. Serodiagnosis of human bocavirus infection, Clin Infect Dis, 46 (2008), pp. 540-546

9. B. Sun, Y. Cai, Y. Li, et al. The nonstructural protein NP1 of human bocavirus 1 induces cell cycle arrest and apoptosis in Hela cells, Virology, 440 (2013), pp. 75-83

10. Y. Luo, A.Y. Chen, J. Qiu. Bocavirus infection induces a DNA damage response that facilitates viral DNA replication and mediates cell death, J Virol, 85 (2011), pp. 133-145

11. Q. Huang, X. Deng, Z. Yan, et al. Establishment of a reverse genetics system for studying human bocavirus in human airway epithelia, PLoS Pathog, 8 (2012

12. Tuomas Jartti, Klaus Hedman, Laura Jartti, Olli Ruuskanen. Human bocavirus the first 5 years, Rev. Med. Virol., 2012; 22:46:64

13. Tobias Allander. Human bocavirus, Journal of Clinical Virology 41 (2008) 29- 33

14. Moriyama Y,. Hamada H., Okada M., et al. Distinctive clinical features of human bocavirus in children younger than 2 years, European Journal of Pediatrics, 2010 169:1087-109

15. Jartti T., Söderlund-Venermo M., Allander T,, Vuorinen T,, Hedman $\mathbf{K}$, Ruuskanen $\mathbf{O}$. No efficacy of prednisolone in acute wheezing associated with human bocavirus infection. Pediatric Infectious Disease Journal, 2011; 30: 521-523.

16. Lee J.I., Chung J.Y., Han T.H., Song M.O., Hwang E.S. Detection of human bocavirus in children hospitalized because of acute gastroenteritis. J Infect Dis 2007

17. Van den Hoogen B.G., de Jong J.C., Groen J., et al. A newly discovered human pneumovirus isolated from young children with respiratory tract disease, Nat Med. 2001; 7(6):719.

18. Swagatika Pandaa, Nirmal Kumar Mohakudb, Lindomar Penac, Subrat Kumara. Human metapneumovirus: review of an important respiratory pathogen, International Journal of Infectious Diseases, Volume 25, August 2014, 45-52

19. Viorel Alexandrescu, Cristina Tecu, Maria-Elena Mihai. Diagnosticul Infectiilor Respiratorii Virale Non-Gripale. Ed. NITECH, Bucuresti 2009, ISBN 978-973-0-07455-0.

\section{Acknowledgment}

This paper is supported by SOP HRD, financed from the European Social Fund and by the Romanian Government under the contract number POSDRU/159/1.5/S/137390

20. J.S. Kahn. Epidemiology of human metapneumovirus, Clin Microbiol Rev, 19 (2006), pp. 546-557

21. J.A. Mullins, D.D. Erdman, G.A. Weinberg, K. Edwards, C.B. Hall, F.J. Walker. Human metapneumovirus infection among children hospitalized with acute respiratory illness, Emerg Infect Dis, 10 (2004), pp. 700-705

22. B.G. Van den Hoogen, J.C. de Jong, J. Groen, T. Kuiken, R. de Groot, R.A. Fouchier, et al. A newly discovered human pneumovirus isolated from young children with respiratory tract disease, Nat Med, 7 (2001), pp. 719-724

23. Hamelin M.E., Prince G.A., Gomez A.M., Kinkead R., Boivin G. Human metapneumovirus infection induces long-term pulmonary inflammation associated with airway obstruction and hyperresponsiveness in mice, J Infect Dis. 2006; 193(12):1634.

24. James E.C., Martin S.H., Sheldon L.K., Anna R.T. Human metapneumovirus infections, Uptodate 2015

25. D. Peng, X. Zhao, E. Liu, Y. Huang, X. Yang, Y. Zhao, et al. Analysis of viral load in children infected with human metapneumovirus, Iran J Pediatr, 20 (2010), pp. 393-400

26. Williams J.V., Harris P.A., Tollefson S.J., et al. Human metapneumovirus and lower respiratory tract disease in otherwise healthy infants and children, N Engl J Med. 2004; 350(5):443.

27. Hamelin M.E., Prince G.A., Boivin G. Effect of ribavirin and glucocorticoid treatment in a mouse model of human metapneumovirus infection. Antimicrob Agents Chemother. 2006; 50(2):774.

28. L. Kitanovski, S. Kopriva, M. Pokorn, M.B. Dolnicar, et al. Treatment of severe human metapneumovirus pneumonia in an immunocompromised child with oral ribavirin and IVIG, J Pediatr Hematol Oncol, 35 (2013), pp. e311-e313

29. Clinical Trials. Gov evaluation of the immunogenicity and safety of a live attenuated human metapneumovirus vaccine. http://clinicaltrials. gov/show/NCT01255410

30. N.D. Ulbrandt, H. Ji, N.K. Patel, J.M. Riggs, Y.A. Brewah, S. Ready, et al. Isolation and characterization of monoclonal antibodies which neutralize human metapneumovirus in vitro and in vivo, $J$ Virol, 80 (2006), pp. 7799-7806

31. M.E. Hamelin, C. Gagnon, G.A. Prince, P. Kiener, J. Suzich, N. Ulbrandt, et al. Prophylactic and therapeutic benefits of a monoclonal antibody against the fusion protein of human metapneumovirus in a mouse model, Antivir Res, 88 (2010), pp. 31-37

32. J. Reina, C. López-Causapé, E. Rojo-Molinero, R. Rubio. Clinico-epidemiological characteristics of acute respiratory infections by coronavirus OC43, NL63 and 229E, Revista Clínica Espanola (English Edition), Volume 214, Issue 9, December 2014, 499-504

33. Kenneth M., Martin H., Anna T. Coronaviruses, Uptodate 2015

34. Nicola Principi, Samantha Bosis, Susanna Esposit. Effects of Coronavirus Infections in Children Eid Journal Volume 16, Number 2-February 2010

35. Vabret A., Dina J., Gouarin S., Petitjean J., Tripey V., Brouard J., Freymuth F. Human (non-severe acute respiratory syndrome) coronavirus infections in hospitalised children in France. J Paediatr Child Health. 2008; 44(4):176. 
36. Kuypers J., Martin E.T., Heugel J., Wright N., Morrow R., Englund J.A. Clinical disease in children associated with newly described coronavirus subtypes. Pediatrics. 2007; 119(1):e70.

37. Woo, PCY; Lau SKP; Yip C. More and More Coronaviruses: Human Coronavirus HKU1 VIRUSES-BASEL, 06/2009, Volume 1, Issue 1

38. Gagneur Arnaud; Vallet, Sophie; Talbot, Pierre J; et al. Outbreaks of human coronavirus in a paediatric and neonatal intensive care unit, European Journal of Pediatrics, 12/2008, Volume 167, Issue 12

39. Arden K.E., M.D. Nissen, T.P. Sloots, M. Mackay. New human coronavirus, HCoV-NL63, associated with severe lower respiratory tract disease in Australia. J Med. Virol. 2005 75:455-462.

40. Keyaerts E., Li S., Vijgen L., et al. Antiviral activity of chloroquine against human coronavirus OC43 infection in newborn mice, Antimicrob Agents Chemother 2009; 53:3416

41. Diane P., J. Owen H., Martin H., Morven E. Epidemiology, clinical manifestations, and pathogenesis of rhinovirus infections, Uptodate 2015

42. Lau S.K., Yip C.C., Tsoi H.W., Lee R.A., So L.Y., Lau Y.L., et al. Clinical features and complete genome characterization of a distinct human rhinovirus (HRV) genetic cluster, probably representing a previously undetected HRV species, human rhinovirus-C, associated with acute respiratory illness in children. J Clin, Microbiol 2007; 45:3655-64

43. Heymann P.W., Platts-Mills T.A., Johnston S.L. Role of viral infections, atopy and antiviral immunity in the etiology of wheezing exacerbations among children and young adults. Pediatr Infect Dis J. 2005; 24(11 Suppl):S217.

44. Miller E.K., Edwards K.M., Weinberg G.A., Iwane M.K., Griffin M.R., Hall C.B., et al. A novel group of rhinoviruses is associated with asthma hospitalizations. J Allergy Clin Immunol 2009; 123:98-104.e1.

45. Bochkov Y.A., Gern J.E. Clinical and molecular features of human rhinovirus C. Microbes Infect. 14: 485-494. (2012)
46. Wei-Ju Chena, John C. Arnoldb, Mary P. Fairchoka et al, Epidemiologic, clinical, and virologic characteristics of human rhinovirus infection among otherwise healthy children and adults: Rhinovirus among adults and children, Journal of Clinical Virology, Volume 64, March 2015, 74-82

47. Holly A. Basta, Jean-Yves Sgro, Ann C. Palmenberg, Modeling of the human rhinovirus $C$ capsid suggests a novel topography with insights on receptor preference and immunogenicity, Virology Volume 448, 5 January 2014, 176-184

48. Lau S.K.P., Yip C.C.Y., Lin A.W.C., Lee R.A., So L.Y., Lau Y.L., et al. Clinical and molecular epidemiology of a novel rhinovirus species, human rhinovirus C. J Infect Dis 2009; 200:1096-103

49. S.K.P. Lau, C.C.Y. Yip, P.C.Y. Woo, K-Y. Yuen. Human rhinovirus C: a newly discovered human rhinovirus species, Emerging Health Threats Journal 2010

50. Cristina Calvo, M. Luz García, Francisco Pozo, et al. Role of Rhinovirus C in Apparently Life-Threatening Events in Infants, Spain EID Journal, Volume15, 9:2009

51. Miller E.K., Edwards K.M., Weinberg G.A., et al. A novel group of rhinoviruses is associated with asthma hospitalizations, J Allergy Clin Immunol. 2009; 123:105-6

52. Tapparel C., L'Huillier A.G., Rougemont A.L., et al. Pneumonia and pericarditis in a child with HRV-C infection: a case report. J Clin Virol 2009; 45:157-60

53. Miller E.K., Lu X., Erdman D.D., Poehling K.A., Zhu Y., Griffin M.R., et al. Rhinovirus-associated hospitalizations in young children, J Infect Dis 2007; 195:773-81

54. Lemanske Jr R.F., Jackson D.J., Gangnon R.E., et al. Rhinovirus illnesses during infancy predict subsequent childhood wheezing, J Allergy Clin Immunol, 2005;116:571-7. 